\title{
On Some Methods for Solution of Linear Diophantine Equations
}

\author{
Azam A. Imomov ${ }^{1 *}$ and Yorqin T. Khodjaev ${ }^{1}$ \\ ${ }^{1}$ Karshi State University, 17 Kuchabag Street, 100180 Karshi City, Uzbekistan \\ ${ }^{*}$ Corresponding author
}

\section{Article Info}

Keywords: Diophantine equation, partial solution, general solution.

2010 AMS: 11D45, $11 D 72$

Received: 2 November 2020

Accepted: 17 June 2020

Available online: 22 June 2020

\begin{abstract}
The paper considers a linear Diophantine equation. A method (algorithm) for finding a general class of solutions of equation is proposed. The proposed algorithm is explained by examples of equations with two and three variables, trying to direct the reader to a general idea that describes the essence of the method used.
\end{abstract}

\section{Introduction}

A Diophantine equation is an equation in several variables in which only integer solutions are allowed. One of its special cases is the linear Diophantine equation in $n \in \mathbb{N}$ variables (where $\mathbb{N}:=\{1,2, \ldots\}$ ), which is of the following general form

$$
A_{1} x_{1}+A_{2} x_{2}+\cdots+A_{n} x_{n}=B,
$$

whose solutions are required to be integers, where $\left\{A_{i}, B\right\} \subset \mathbb{Z}, n \geq 2$ and $i=1,2, \ldots, n$, where $\mathbb{Z}$ is the set of all integers. Equation (1.1) is named in honor of the Greek mathematician Diophantus of Alexandria (circa 300 c.e.).

A large number of works on the solution of Linear Diophantine equations are devoted. In these works, various methods and algorithms for solving equations are proposed and developed. For instance in [4], methods based on arguments of Euclidean algorithm are proposed. In this regard, we refer the reader, also to [1], [2], [3], [5], [6].

The aim of the paper is to attempt to give a general algorithm for finding the class of all solutions of equation (1.1), which, unlike the mentioned methods, would simplify the process of finding solutions.

In the second section, we give an algorithm for the method of finding the class of all solutions of an equation with two variables. Moreover, we will explain this algorithm using a typical example, trying to direct the reader to a general idea that describes the essence of the proposed method. In fact, the algorithm we proposed in this case, is based on arguments that differ significantly from the Euclidean algorithm. The latter is known as the algorithm for finding the greatest common divisor of integers.

In the Third and Fourth sections, the developed algorithm for an equation with two variables extends to cases of an equation with three and more variables.

\section{Equation with two variables}

Let $n=2$ in equation (1.1). Then we consider the following equation with two variables $x$ and $y$ :

$$
A x+B y=C,
$$

where $\{A, B, C\} \subset \mathbb{Z}$ are the given numbers. The greatest common divisor of numbers $|A|$ and $|B|$ is denote by gcd $(|A|,|B|)$. There are many sources devoted to finding solutions to equations (2.1). It is known that if the number $|C|$ is not divisible by $\operatorname{gcd}(|A|,|B|)$, then equation $(2.1)$ has no solutions; see [4].

Now consider the case that the number $C$ is divisible by $\operatorname{gcd}(|A|,|B|)$. Let's divide numbers $A, B$, and $C$ by $\operatorname{gcd}(|A|,|B|)$. Then equation $(2.1)$ be transformed to the following form:

$$
a x+b y=c,
$$


where the numbers $a, b$, and $c$ don't have a common divisor, that is $\operatorname{gcd}(a, b, c)=1$. Integers with this property are hereinafter referred to as prime numbers in common.

It is known that to construct all the solutions of equation (2.2), one partial solution is needed.

\subsection{General Algorithm for finding a partial solution.}

As this is often done in order to find all solutions of equation (2.2), it is first necessary to somehow establish one partial solution. Typically, such a solution can be found primitively, using intuitive consideration. Then, using this solution, it is easy to construct the class of all solutions. In this case, a specific analytical method for finding a partial solution is not used. In this section, we put forward one algorithm for the method of finding a partial solution, based in fact, on the arguments essentially differing from Euclidian algorithm. Namely our algorithm is based on a very simple but a very important for our aim idea: searching for a linear representation of the unit using a finite number of so-called superpositions of the coefficients of the equation in question. And also, as will be shown below, this algorithm is applicable for cases of equations with an arbitrary number of variables.

Definition 2.1. The number obtained as a result of eventual operations, which consist of the algebraic actions of addition and subtraction between several prime numbers in common, we call the Superposition of these numbers.

Let

$$
\mathbb{D}:=\left\{a_{1}, a_{2}, \ldots, a_{k}\right\} \subset \mathbb{Z}, \quad k \in \mathbb{N} .
$$

Henceforth we denote

$$
\operatorname{Spos}(\mathbb{D}) \quad \text { or } \operatorname{Spos}\left(a_{i_{1}}, a_{i_{2}}, \ldots, a_{i_{j}}\right), j \leq k,
$$

any superposition of numbers from the set $\mathbb{D}$, where $a_{i_{m}} \in \mathbb{D}, i_{m} \in \mathbb{N}$ and $m=1,2, \ldots, j$.

The essence of the proposed algorithm will be based on the following simple axiom that describes the elementary properties of integers.

Main Axiom 2.1. Using repeatedly superposition of two mutually prime numbers, one can construct any integer.

First, we give an example.

Exercise 2.2. Find a partial solution of the equation

$$
127 x+36 y=79 .
$$

Solution. The coefficients of the equation $a=127, b=36$, as seen, are mutual prime numbers. According to the Main axiom, using a superposition of the same numbers we find, for example, the following number:

1) $\operatorname{Spos}(a, b)=a-(b+b+b)=a-3 b=127-3 \times 36=19=: a_{1}$.

Let continue to construct superpositions using the coefficients $a, b$ and $a_{1}$ :

2) $\operatorname{Spos}\left(a, a_{1}\right)=a-5 a_{1}=-4 a+15 b=32=: a_{2}$;

3) $\operatorname{Spos}\left(a_{1}, a_{2}\right)=a_{2}-a_{1}=-5 a+18 b=13=: a_{3}$;

4) $\operatorname{Spos}\left(a_{1}, a_{3}\right)=a_{1}-a_{3}=6 a-21 b=6=: a_{4}$;

5) $\operatorname{Spos}\left(a_{3}, a_{4}\right)=a_{3}-2 a_{4}=-17 a+60 b=1$.

In the last step, we built the number 1 as follows:

$$
-17 a+60 b=1 .
$$

Multiplying both sides of equality (2.4) by 79, we can easily get one solution to the equation (2.3). In fact, this follows from (2.4) that

$$
-1343 a+4740 b=79,
$$

so that $\left(x_{0}=-1343 ; y_{0}=4740\right)$ is the partial solution of equation $(2.3)$.

Note that we built the superpositions so that the values of the sequence of $\left\{a_{k}, k \in \mathbb{N}\right\}$ step by step approached unity, and continued the process until they reached unity. It reached in step 5. In other words, we "crushed" the right side of equation (2.3) and as a result we got equality (2.4). Based on what has been done, we call the proposed method the Crushing method.

We also note two more moments. Firstly, the set of steps is not unique, because superpositions of two numbers can be constructed as many time as desired, and the number of steps to the final equality of type (2.4) depends on the choice of these superpositions. Secondly, as a consequence of the first moment, the partial solution $\left(x=x_{0} ; y=y_{0}\right)$ may be completely different.

The proposed Crushing method for finding one (partial) solution to equation (2.3) is universal in the sense that for any equation of type (3), a finite number of superposition steps can be performed to crush up the right-hand side to unity and thereby obtain an equality of type (2.4). In fact, for given coefficients $\{a, b, c\} \subset \mathbb{Z}$, one can always find the minimum number of steps of such superpositions.

Now we give a general description of our algorithm. We follow the procedure from the solution of equation (2.3). Let it be required to find one partial solution of equation (2.2) with the set of coefficients $\mathbb{D}_{1}:=\{a, b\} \subset \mathbb{Z}$. The following sequence of superpositions will be performed, until an appearance of the number 1:

$$
\begin{aligned}
& \operatorname{Spos}\left(\mathbb{D}_{1}\right)=: a_{1} \text { and define } \mathbb{D}_{2}:=\left\{\mathbb{D}_{1}, a_{1}\right\} ; \\
& \operatorname{Spos}\left(\mathbb{D}_{2}\right)=: a_{2} \text { and define } \mathbb{D}_{3}:=\left\{\mathbb{D}_{2}, a_{2}\right\} ; \\
& \ldots \quad \ldots \quad \ldots \quad \ldots \quad \ldots \quad \ldots \quad \ldots \cdots \\
& \operatorname{Spos}\left(\mathbb{D}_{k}\right)=1 \text { for some } k \in \mathbb{N},
\end{aligned}
$$


where $\mathbb{D}_{k}:=\left\{\mathbb{D}_{k-1}, a_{k-1}\right\}$. From last equality we obtain

$$
c \cdot \operatorname{Spos}\left(\mathbb{D}_{k}\right)=a x_{0}+b y_{0}=c,
$$

and needless to say we get $\left(x_{0} ; y_{0}\right)$ - the solution of equation (2.3).

Thus, the above algorithm (Crushing method) can be admitted as a General Algorithm for finding a partial solution to the equation with two variables of the form (2.2).

\subsection{General class of solutions.}

Suppose that one pair of solutions $\left(x_{0} ; y_{0}\right)$ is known. As we proved in the previous subsection, for equations that have a solution, one can always find one pair of solutions by using the Crushing method. Then it is obvious that equation (2.2) can be written as

$$
a x+b y=a x_{0}+b y_{0} .
$$

Hence

$$
a\left(x-x_{0}\right)=b\left(y_{0}-y\right)
$$

Since $\operatorname{gcd}(a, b)=1$, it is necessary to be $x-x_{0}=b k$, where $k \in \mathbb{Z}$. Then the general solution of equation (2.2) will have the following form:

$$
\left\{\begin{array}{l}
x=x_{0}+b k \\
y=y_{0}-a k
\end{array}\right.
$$

In view of the found general solution (2.6), we can write the equation (2.2) in the following equivalent form:

$$
a x+b y=a\left(x_{0}+b k\right)+b\left(y_{0}-a k\right),
$$

and therefore

$$
a\left(x-x_{0}\right)+b\left(y-y_{0}\right)-a b k+a b k=0 .
$$

Relation (2.8) shows that equations (2.5) and (2.7) are the same. From here one can get a solution in the form of $x=x_{0}-b k$ and $y=y_{0}+a k$. Therefore, the general solution is finally written in the following form:

$$
\left\{\begin{array}{l}
x=x_{0} \pm b k \\
y=y_{0} \mp a k
\end{array}\right.
$$

where $k$ is any number of $\mathbb{Z}$. From the last reasoning, we can conclude that in order to obtain a general solution to equation (2.5), it is sufficient to rewrite the equation (2.5) moving all expressions to the left-hand side and add zero in the form

$$
-a b k+a b k
$$

In particular, according to (2.9), we obtain the general solution of equation (2.3) in the following form:

$$
\left\{\begin{array}{l}
x=-1343 \pm 36 k, \\
y=4740 \mp 127 k,
\end{array}\right.
$$

where $k$ is any number of $\mathbb{Z}$. Note that a particular solution can be minimized by choosing $\mathrm{k}$. In this case, the general class of solutions of equation (2.3) can be written more simply:

$$
\left\{\begin{array}{l}
x=25 \pm 36 k \\
y=-86 \mp 127 k .
\end{array}\right.
$$

\section{Equation with three variables}

In this section, we demonstrate a solution to an equation with three unknowns. Without loss of generality, as in the case of two variables, we consider the equation

$$
a x+b y+c z=d,
$$

where $\{a, b, c, d\} \subset \mathbb{Z}$ and $\operatorname{gcd}(a, b, c)=1$. 


\subsection{Crushing method for three variables.}

The previous section describes a general algorithm for finding one partial solution to the equation with two variables using the so-called Crushing method. Here we demonstrate the possibility of spreading this method to solve the equation (3.1). The concept of a superposition of three or more prime numbers in common is defined similarly to the case with two numbers. Further, we use the following statement, the proof of which follows from the Main Axiom.

Proposition 3.1. Using the repeat superposition of three or more prime numbers in common, one can construct any integer.

Exercise 3.2. Find a partial solution of the equation

$$
30 x+105 y+56 z=13 .
$$

Solution. The set coefficients of (3.2) is $\{a, b, c\}=\{30,105,56\}$. By virtue of Proposition above, using the superposition of these coefficients, we make the following steps:

1) $\operatorname{Spos}(a, c)=2 a-c=4=: a_{1}$;

2) $\operatorname{Spos}\left(b, a_{1}\right)=b-26 a_{1}=b-52 a+26 c=1$.

We have constructed number 1 only in the second step:

$$
-52 a+b+26 c=1 .
$$

Multiplying both sides of the last equality by 13, we obtain

$$
-676 a+13 b+338 c=13,
$$

and thereby we found one (partial) solution of equation (3.2):

$$
x_{0}=-676, \quad y_{0}=13, \quad z_{0}=338 .
$$

The general class of solutions will be constructed below.

As in the case of two variables, we note that there will be no obstacles to constructing the minimum number of steps of the corresponding superpositions for any given set of coefficients $\{a, b, c, d\} \subset \mathbb{Z}$ in equation (3.1), as a result of which we achieve one partial solution.

\subsection{General class of solutions.}

As noted in the previous subsection, for equations that have solutions, one can always find one solution $\left(x_{0} ; y_{0} ; z_{0}\right)$ using the crushing method Now, using this solution, we intend to obtain a class of all solutions. For this, we extend the method from previous subsection to the case under consideration. Similarly to equality (2.8), we write the equation (3.1) in the following form:

$$
a\left(x-x_{0}\right)+b\left(y-y_{0}\right)+c\left(z-z_{0}\right)-a b k_{1}+a b k_{1}-a c k_{2}+a c k_{2}-c b k_{3}+c b k_{3}=0 .
$$

Therefore, we can present the general solution in the form

$$
\left\{\begin{array}{l}
x=x_{0} \pm b k_{1} \pm c k_{2}, \\
y=y_{0} \mp a k_{1} \pm c k_{3}, \\
z=z_{0} \mp a k_{2} \mp b k_{3},
\end{array}\right.
$$

where $\left\{k_{1}, k_{2}, k_{3}\right\} \subset \mathbb{Z}$.

The following matrix clearly indicates to the coefficients and their signs in each row of the system (3.4). Here, in front of the matrix, on the column we put the partial solution by the corresponding variable, on the rows of the matrix are the coefficients in the order specified in the equation (3.1).

$$
\begin{aligned}
& x_{0} \longleftrightarrow \\
& y_{0} \longleftrightarrow \\
& z_{0} \longleftrightarrow
\end{aligned}\left[\begin{array}{ccc}
\mathbf{0} \cdot a & \pm b & \pm c \\
\mp a & \mathbf{0} \cdot b & \pm c \\
\mp a & \mp b & \mathbf{0} \cdot c
\end{array}\right] .
$$

In each row of the matrix, multiplication by zero indicates the absence of the corresponding coefficient. For example, in the second row there is no second coefficient, which is consistent with the second row of system (3.4). The coefficients multiplied by zero form the diagonal of the matrix, which divides it into two parts. Coefficients in the upper part of the diagonal, as a rule, have a plus sign, and in the lower part coefficients with a minus sign.

The constructed matrix which we call the matrix of solution class, visually specifies the form of the general solution of the equations in question.

In particular, based on (3.4) and (3.5), we can construct the class of general solutions of equation (3.2) in the following form:

$$
\left\{\begin{array}{l}
x=676 \pm 105 k_{1} \pm 56 k_{2}, \\
y=13 \mp 30 k_{1} \pm 56 k_{3}, \\
z=338 \mp 30 k_{2} \mp 105 k_{3},
\end{array}\right.
$$

where $\left\{k_{1}, k_{2}, k_{3}\right\}$ is any set inside $\mathbb{Z}$.

Remark 3.3. In the book [4, pp. 23-33] proposed an algorithm for finding a solution of linear Diophantine equations, which requires a fairly lengthy process. The application of this algorithm was demonstrated by the example of one equation with three variables. By comparing, we can make sure that our proposed method looks slightly simpler. 


\section{Equation with $n$ variables}

Now consider the equation (1.1) with $n$ variables. Dividing by $\operatorname{gcd}\left(A_{1}, A_{2}, \ldots, A_{n}\right)$, we write it in the form

$$
a_{1} x_{1}+a_{2} x_{2}+\cdots+a_{n} x_{n}=b,
$$

where $\left\{a_{i}, b\right\} \subset \mathbb{Z}, i=1,2, \ldots, n$ and $\operatorname{gcd}\left(a_{1}, a_{2}, \ldots, a_{n}\right)=1$.

\subsection{General class of solutions.}

In this subsection, we demonstrate a general description of the algorithm for constructing the class of all solutions of equation (4.1). Suppose that the set of numbers $\left(\alpha_{1}, \alpha_{2}, \ldots, \alpha_{n}\right)$ is the partial solution to this equation. Such a solution can always be found using the general algorithm (crushing method) described in the previous sections. To find a general solution to the equation, we represent it in the form of type (3.3). After similar reasoning as in Subsection 3.2, we can construct the class of all solutions of equation (4.1) in the following form:

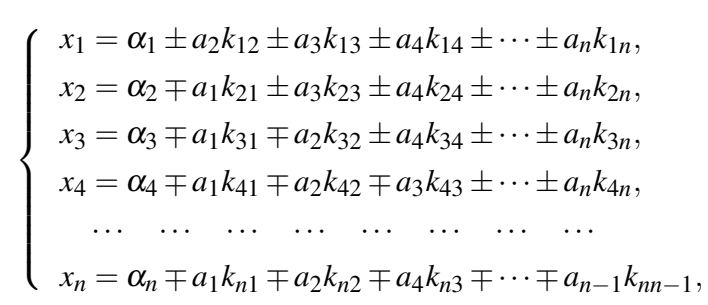

where $\left\|k_{i j}\right\|_{i, j=1}^{n}$ is the quadratic matrix such that $k_{i j} \in \mathbb{Z}, k_{i i}=0$ and $k_{i j}=k_{j i}$ for all $i, j=1,2, \ldots, n$.

The following matrix, as in the case of three variables, gives a clear picture of constructing a class of general solutions of the equation, indicating the coefficients and their signs in each row of the system (4.2):

$$
\begin{gathered}
\alpha_{1} \longleftrightarrow \\
\alpha_{2} \longleftrightarrow \\
\alpha_{3} \longleftrightarrow \\
\vdots \\
\alpha_{n-1} \longleftrightarrow \\
\alpha_{n} \longleftrightarrow
\end{gathered}\left[\begin{array}{rrrrrc}
\mathbf{0} \cdot a_{1} & \pm a_{2} & \pm a_{3} & \cdots & \pm a_{n-1} & \pm a_{n} \\
\mp a_{1} & \mathbf{0} \cdot a_{2} & \pm a_{3} & \cdots & \pm a_{n-1} & \pm a_{n} \\
\mp a_{1} & \mp a_{2} & \mathbf{0} \cdot a_{3} & \cdots & \pm a_{n-1} & \pm a_{n} \\
\vdots & \vdots & \vdots & \ddots & \vdots & \vdots \\
\mp a_{1} & \mp a_{2} & \mp a_{3} & \cdots & \mathbf{0} \cdot a_{n-1} & \pm a_{n} \\
\mp a_{1} & \mp a_{2} & \mp a_{3} & \cdots & \mp a_{n-1} & \mathbf{0} \cdot a_{n}
\end{array}\right]
$$

Note that in the case of an equation with many variables, constructing the matrix of the form (4.3) greatly simplifies discussions in solving the equations.

Remark 4.1. According to the fact that $k_{i j}=k_{j i}, i, j=1,2, \ldots, n$, the number of all different symbols $k_{i j}$ is $\left(\begin{array}{l}n \\ 2\end{array}\right)$, i.e.

$$
\left(\begin{array}{l}
n \\
2
\end{array}\right)=\frac{n(n-1)}{2} .
$$

Let's get to the examples.

Exercise 4.2. Find a partial solution of the equation

$$
30 x+42 y+105 z+70 u=17 .
$$

Solution. The coefficients of the equation are $a=30, b=42, c=105$ and $d=70$. Using the superpositions of the coefficients we obtain

1) $\operatorname{Spos}(b, d)=2 b-d=14=: a_{1}$;

2) $\operatorname{Spos}\left(a, a_{1}\right)=a-2 a_{1}=a-4 b+2 d=2=: a_{2}$;

3) $\operatorname{Spos}\left(c, a_{2}\right)=c-52 a_{2}=c-52 a+208 b-104 d=1$.

We obtained

$$
-52 a+208 b+c-104 d=1 .
$$

Therefore

$$
-884 a+3536 b+17 c-1768 d=17,
$$

and thus we have a partial solution

$$
x_{0}=-884, \quad y_{0}=3536, \quad z_{0}=17, \quad u_{0}=-1768 .
$$

Now we construct the matrix of solution class:

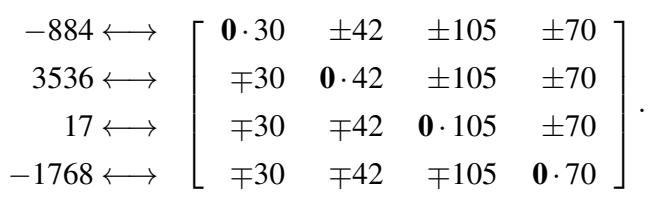


Thereafter we can construct the class of all solutions in the form

$$
\left\{\begin{array}{l}
x=-884 \pm 42 k_{12} \pm 105 k_{13} \pm 70 k_{14}, \\
y=3536 \mp 30 k_{21} \pm 105 k_{23} \pm 70 k_{24}, \\
z=17 \mp 30 k_{31} \mp 42 k_{32} \pm 70 k_{34}, \\
u=-1768 \mp 30 k_{41} \mp 42 k_{42} \mp 105 k_{43} .
\end{array}\right.
$$

This system is a class of general solutions of equation (4.5) in accordance with the representation (4.2). Moreover, due to (4.4), the form of the general solution can be simplified by getting rid of double indices in numbers $k_{i j}$ :

$$
\left\{\begin{array}{l}
x=-884 \pm 42 k_{1} \pm 105 k_{2} \pm 70 k_{3}, \\
y=3536 \mp 30 k_{1} \pm 105 k_{4} \pm 70 k_{5}, \\
z=17 \mp 30 k_{2} \mp 42 k_{4} \pm 70 k_{6}, \\
u=-1768 \mp 30 k_{3} \mp 42 k_{5} \mp 105 k_{6},
\end{array}\right.
$$

where $\left\{k_{i}, i=1,2, \ldots, 6\right\} \subset \mathbb{Z}$.

\subsection{Special cases.}

As in many mathematical theories, we can separate some cases related to the method of searching for partial solutions. In the following subsections, we highlight several special cases for which there is no need to use a general algorithm to find the partial solution.

\subsection{1.}

If in the equation $b=0$, then the class of its general solutions can be constructed very simply, as in the following example.

Exercise 4.3. Find a class of general solutions of the equation

$$
96 x+11 y+75 z+8 u+31 v=0 .
$$

Solution. It is seen that $x=y=z=u=v=0$ is one of the partial solutions. By using (4.3), let's build the matrix

$$
\begin{aligned}
& 0 \longleftrightarrow \\
& 0 \longleftrightarrow \\
& 0 \longleftrightarrow \\
& 0 \longleftrightarrow
\end{aligned}\left[\begin{array}{rrrrr}
\mathbf{0} \cdot 96 & \pm 11 & \pm 75 & \pm 8 & \pm 31 \\
\mp 96 & \mathbf{0} \cdot 11 & \pm 75 & \pm 8 & \pm 31 \\
\mp 96 & \mp 11 & \mathbf{0} \cdot 75 & \pm 8 & \pm 31 \\
\mp 96 & \mp 11 & \mp 75 & \mathbf{0} \cdot 8 & \pm 31 \\
\mp 96 & \mp 11 & \mp 75 & \mp 8 & \mathbf{0} \cdot 31
\end{array}\right]
$$

Then the class of general solutions to the equation can be constructed in the following form:

$$
\left\{\begin{array}{l}
x=0 \pm 11 k_{12} \pm 75 k_{13} \pm 8 k_{14} \pm 31 k_{15} \\
y=0 \mp 96 k_{21} \pm 75 k_{23} \pm 8 k_{24} \pm 31 k_{25} \\
z=0 \mp 96 k_{31} \mp 11 k_{32} \pm 8 k_{34} \pm 31 k_{35} \\
u=0 \mp 96 k_{41} \mp 11 k_{42} \mp 75 k_{43} \pm 31 k_{45} \\
v=0 \mp 96 k_{51} \mp 11 k_{52} \mp 75 k_{53} \mp 8 k_{54}
\end{array}\right.
$$

where numbers $k_{i j} \in \mathbb{Z}$, such that $k_{i i}=0$ and $k_{i j}=k_{j i}$ for all $i, j=1,2, \ldots, 5$.

\subsection{2.}

If the number $b$ is divisible by some one of the coefficients $\left\{a_{1}, a_{2}, \ldots, a_{n}\right\}$, then the equation can be solved as in the following example.

Exercise 4.4. Find a class of general solutions of the equation

$$
96 x+11 y+75 z+3 u+31 v=27 .
$$

Solution. We can put $x=y=z=v=0$ and get $u=9$. Hence by using (4.3), we obtain

$$
\left\{\begin{array}{l}
x=0 \pm 11 k_{12} \pm 75 k_{13} \pm 3 k_{14} \pm 31 k_{15} \\
y=0 \mp 96 k_{21} \pm 75 k_{23} \pm 3 k_{24} \pm 31 k_{25} \\
z=0 \mp 96 k_{31} \mp 11 k_{32} \pm 3 k_{34} \pm 31 k_{35} \\
u=9 \mp 96 k_{41} \mp 11 k_{42} \mp 75 k_{43} \pm 31 k_{45} \\
v=0 \mp 96 k_{51} \mp 11 k_{52} \mp 75 k_{53} \mp 3 k_{54}
\end{array}\right.
$$

herein $k_{i j} \in \mathbb{Z}$, such that $k_{i i}=0$ and $k_{i j}=k_{j i}$ for all $i, j=1,2, \ldots, 5$. 


\subsection{3.}

If the set of coefficients $\left\{a_{1}, a_{2}, \ldots, a_{n}\right\}$ contains a pair of coefficients $\left\{a_{i 1}, a_{i 2}\right\}$ such that $\operatorname{gcd}\left(a_{i 1}, a_{i 2}\right)=1$, then the class of general solutions of equation (4.1) can be constructed based on the solution of the equation with these coefficients and with the corresponding variables.

Exercise 4.5. Find a class of general solutions of the equation

$$
96 x+11 y+75 z+8 u+31 v=27 .
$$

Solution. Putting $x=z=v=0$ we have

$$
11 y+8 u=27 .
$$

Now the solution to the last equation does not seem complicated. Thence we can easily construct the class of general solutions in the form of

$$
\left\{\begin{array}{l}
x=0 \pm 11 k_{12} \pm 75 k_{13} \pm 8 k_{14} \pm 31 k_{15}, \\
y=9 \mp 96 k_{21} \pm 75 k_{23} \pm 8 k_{24} \pm 31 k_{25}, \\
z=0 \mp 96 k_{31} \mp 11 k_{32} \pm 8 k_{34} \pm 31 k_{35}, \\
u=-9 \mp 96 k_{41} \mp 11 k_{42} \mp 75 k_{43} \pm 31 k_{45}, \\
v=0 \mp 96 k_{51} \mp 11 k_{52} \mp 75 k_{53} \mp 8 k_{54},
\end{array}\right.
$$

where numbers $k_{i j} \in \mathbb{Z}$ are as above.

Remark 4.6. When it is necessary, as in Example 4.5, that the class of general solutions of equation (4.1) can be constructed based on the solution of the equation with three variables, if the set of coefficients $\left\{a_{1}, a_{2}, \ldots, a_{n}\right\}$ contains the subset $\left\{a_{i 1}, a_{i 2}, a_{i 3}\right\}$ for which $\operatorname{gcd}\left(a_{i 1}, a_{i 2}, a_{i 3}\right)=1$.

\section{Concluding notes}

In our opinion, the method proposed in this article is very simple and convenient for use by mathematicians with minimal mathematical skills. We believe that the Crushing method can be developed for a more general case. Subsequently, it will be possible to develop simplified solution methods for nonlinear equations.

So, in our subsequent researches, the proposed method will be modified and applied in other cases. We will pay special attention to minimizing the amount of numbers $k_{i j} \in \mathbb{Z}$. In particular, we will use it when searching for a solution to a system of linear Diophantine equations.

As an example, without details, here we give a general solution to the following system of equations found using the algorithm proposed above:

$$
\left\{\begin{array}{l}
3 x+4 y=2 \\
5 x+2 z=12 \\
y+3 u=11
\end{array}\right.
$$

Using the algorithm, we can find the solution in the following form:

$$
\left\{\begin{array}{l}
x=2+12 k, \\
y=-1-9 k, \\
z=1-30 k, \\
u=4-4 k,
\end{array}\right.
$$

where $k \in \mathbb{Z}$.

In addition, we will be interested in solvability in integers of the following equation:

$$
\left(a_{1} x_{1}+a_{2} x_{2}+\cdots+a_{n} x_{n}\right)^{p}=b_{1} x_{1}+b_{2} x_{2}+\cdots+b_{n} x_{n},
$$

where $p \in \mathbb{N}$ and $\left\{a_{i}, b_{i}\right\} \subset \mathbb{Z}, i=1,2, \ldots, n$.

\section{Acknowledgement}

The authors thank the anonymous reviewer for carefully reading the manuscript and for the kind comments that contributed to the improvement of the paper.

\section{References}

[1] M. Clausen, A. Fortenbacher, Efficient solution of linear diophantine equations, J. Sym. Comp., 8(1-2) (1989), 201-216.

[2] E. Contejean, H. Contejean, An Efficient Incremental Algorithm for Solving Systems of Linear Diophantine Equations, Inf. Comp., 113(1) (1994), $143-172$

[3] H. Esmaeili, How can we solve a linear Diophantine equation by the basis reduction algorithm, Int. J. Comp. Math., 82(10)(2005), 1227-1234.

[4] V. Y. Nesterenko, Number Theory. "Academy", Moscow (in Russian), 2008.

[5] D. Papp, B. Vizvari, Effective solution of linear Diophantine equation systems with an application in chemistry, J. Math. Chem., 39(1)(2006), 15-31.

[6] A. Vigneron-Tenorio, Semigroup ideals and linear diophantine equations, Linear Algebra Appl., 295(1999), 133-144 